\title{
AVALIAÇÃo DO EFEITO DA URBANIZAÇÃo NA PRODUÇÃo DE SEDIMENTOS DA BACIA DO RIO JACARECICA/AL MEDIANTE USO DE MODELO HIDROSSEDIMENTOLÓGICO DISTRIBUIIDO(1)
}

\author{
Samuellson Lopes Cabral ${ }^{(2)}$, Rosangela Sampaio Reis ${ }^{(3)}$ \& Carlos Ruberto Fragoso Júnior ${ }^{(3)}$
}

\section{RESUMO}

A urbanização sem planejamento ao longo da bacia do rio Jacarecica, em Maceió, AL, causa grandes impactos ambientais. A substituição de áreas vegetadas por áreas urbanizadas agrava esses impactos. No entanto, o potencial efeito dessas modificações na produção de sedimentos é desconhecido. Neste trabalho, avaliaram-se mudanças da produção de sedimentos na bacia do rio Jacarecica, no ano de 2010, em razão da alteração de parte da cobertura do solo por urbanização, por meio de modelagem matemática. Os resultados evidenciaram que o mês com maior produção produziu mais de $120 \mathrm{t} / \mathrm{ha} / \mathrm{mês}$, perfazendo o total de $131 \mathrm{t}$, correspondente a uma área de $24,5 \mathrm{~km}^{2}$ da área total da bacia, revelando que grande parte da bacia é realmente susceptível ao processo de erosão. Este trabalho apresenta que a aplicação do modelo KINEROS2 é viável e o seu acoplamento ao SIG é de grande valia para identificação e análise das principais áreas de produção de sedimentos na bacia deste rio, podendo ser considerado ferramenta promissora para simulação da produção de sedimentos em bacias hidrográficas do nordeste brasileiro.

Termos de Indexação: usos do solo, KINEROS2, modelo físico, bacia Jacarecica.

\section{SUMMARY: POTENTIAL EFFECT OF URBANIZATION ON SEDIMENT YIELD BY THE USE OF A HYDRO-SEDIMENTOLOGICAL MODEL COUPLED TO GIS}

Unplanned urbanization along the Jacarecica river basin in Maceió (AL), causes major environmental issues. The substitution of vegetated by urban areas reinforces these impacts.

(1) Parte da Dissertação de Mestrado do primeiro autor apresentada ao Programa de Pós-Graduação em Recursos Hídricos e Saneamento da Universidade Federal de Alagoas - UFAL. Recebido para publicação em 11 de maio de 2012 e aprovado em 17 de abril de 2013.

(2) Doutorando do Programa de Pós Graduação em Engenharia Civil (Recursos Hídricos) pela Universidade Federal do Ceará. Campus Pici. CEP 60451-970 Fortaleza (CE), Brasil. E-mail: samuellsoncabral@hotmail.com

(3) Professores do Programa de Pós Graduação em Recursos Hídricos e Saneamento da Universidade Federal de Alagoas - UFAL. Campus A. C. Simões. CEP 57072-970 Maceió (AL), Brasil. E-mail: rosangelareis_al@hotmail.com, crubertofj@hotmail.com 


\begin{abstract}
However, the potential effect of these changes on the sediment yield is unknown. In this work, we evaluated changes in the sediment yield of the Jacarecica river basin in 2010, caused by changes in the soil cover due to urbanization using mathematical modeling. The results showed that the month with the highest production yielded more than $120 \mathrm{t} / \mathrm{ha} / \mathrm{month}$ of sediment, totaling $131 \mathrm{t}$, corresponding to an area of $24.5 \mathrm{~km}^{2}$ of the total basin area, thus revealing that a great part of the basin is actually susceptible to erosion processes. This study points out that the application of the KINEROS2 model is feasible, and when associated with a geographical information system (GIS), it is useful to identify and analyze the major sediment production areas of this river basin. The tool is therefore considered promising for the simulation of sediment production in watersheds in northeastern Brazil.
\end{abstract}

Index terms: land uses, KINEROS2, physical model, Jacarecica basin.

\section{INTRODUÇÃO}

Recentemente, modelos hidrossedimentológicos de base física vêm sendo cada vez mais aplicados na medição e estimativa da produção de sedimentos em escalas cada vez maiores (Figueiredo \& Bathurst, 2002), trazendo significativas contribuições para a gestão dos recursos hídricos.

Atualmente, o conhecimento dos processos hidrológicos e sedimentológicos em pequenas bacias hidrográficas urbanas também passou a ser importante, uma vez que a utilização dos recursos hídricos disponíveis cresceu juntamente com a população, pois a urbanização provoca modificação substancial no ciclo hidrológico. A disponibilidade de séries de dados medidos de vazões, bem como a avaliação de transporte de sedimentos, de pequenas e médias bacias hidrográficas é muito pequena, com exceção de poucos trabalhos de pesquisa, mas de duração limitada.

A modelagem dos escoamentos urbanos é complexa em razão de diversos fatores, como: diversidade da ocupação urbana, inexistência de cadastramento atualizado da rede de micro- drenagem, interfaces entre o escoamento da superfície e os escoamentos em redes de canais subterrâneos de drenagem, mudanças sucessivas de regimes de escoamento, presença de resíduos sólidos e de sedimentos no escoamento. $\mathrm{O}$ aperfeiçoamento de técnicas de modelagem e os esforços para a quantificação e redução de incertezas nesse campo constituem-se, portanto, em elementos relevantes de desenvolvimento tecnológico.

Os modelos hidrossedimentológicos fornecem estimativas que podem auxiliar a suprir a falta de informações sobre o aporte de sedimentos em bacias hidrográficas. Porém, quando dados medidos são confrontados com os resultados obtidos a partir de modelos, grandes discrepâncias são encontradas (Paiva \& Paiva, 2003). Essa realidade indica a importância de se ampliar a quantidade dados obtidos no campo, no sentido de fornecer essas informações como entrada e auxiliar no aprimoramento e calibração de métodos e modelos de estimativa do transporte de sedimentos. A erosão é importante questão ambiental que interfere diretamente na sociedade, pois reduz a capacidade de reservatórios, intensifica o transporte de poluentes agregados às partículas de sedimentos (Araújo \& Knight, 2005) e diminui o potencial da produção agrícola em áreas agricultáveis, em razão dos processos de desprendimento e arraste de partículas (Firouzabadi \& Davoodi, 2004).

Segundo Cabral (2011), a bacia do rio Jacarecica sofre com processos de degradação desde a nascente até a sua foz, observando-se a ocorrência de urbanização sem planejamento, com ocupação de encostas. A erosão dos talvegues ocorre pela concentração do escoamento, em regiões de alta declividade, e pela falta de proteção das superfícies, contribuindo para o assoreamento da foz. O crescimento desordenado da cidade ao longo da parte alta da bacia é outro fator de grande impacto ambiental.

Este estudo teve como objetivo quantificar, temporal e espacialmente, o regime hidrossedimentológico de produção de sedimentos na bacia do rio Jacarecica, por meio do acoplamento entre o modelo hidrossedimentológico de base física KINEROS2 e da ferramenta hidrológica AGWA2, para ajudar na identificação e representação espacial das áreas mais susceptíveis à erosão.

\section{MATERIAL E MÉTODOS}

\section{Caracterização da Área de Estudo}

A área da bacia hidrográfica do rio Jacarecica está localizada no nordeste do Brasil, na cidade de Maceió, Estado de Alagoas (Figura 1). Trata-se de uma bacia relativamente pequena, que drena uma área de aproximadamente $24,5 \mathrm{~km}^{2}$. O rio principal tem mais ou menos $13 \mathrm{~km}$ de extensão. A pluviosidade média anual é de $1.654 \mathrm{~mm}$.

\section{Dados do Modelo AGWA/KINEROS2}

\section{Modelo numérico de terreno (MNT)}

O MNT da bacia do rio Jacarecica foi gerado com base na carta topográfica da Prefeitura Municipal de Maceió, com uma escala de 1:2.000. Os pontos foram interpolados para gerar um grid com células de $10 \mathrm{~m}$ de resolução. A interpolação adotada foi o método do inverso da distância. 


\section{Uso e cobertura do solo}

Utilizou-se imagem do satélite LANDSAT 5 do sensor Thematic Mapper (TM), em composição Red, Green and Blue (RGB), datada de 08/01/2010, às $12 \mathrm{~h} 15$, horário local, na órbita 214 e ponto 67 , que foi utilizada neste trabalho para classificação da cobertura, conforme uso do solo.

A partir do software de Processamento Digital de Imagens utilizado - ERDAS 9.1, foi realizada a classificação supervisionada. Foram identificadas três classes de cobertura de solo: vegetação, área urbana e solo exposto.

\section{Tipos de solo}

Nessa etapa da modelagem, optou-se por utilizar os dados de tipos de solojá disponíveis pelo AGWA2. Para utilização em regiões fora do território dos EUA, a opção que o AGWA2 fornece corresponde ao mapa de solo da FAO, dado esse utilizado na bacia do rio Jacarecica.

\section{Precipitação pluvial}

Neste estudo, foram utilizados dados de precipitação pluvial dos postos do INMET e da SEMARH, localizados em áreas de influencia da bacia do Rio Jacarecica. No quadro 1, encontram-se as coordenadas dos postos pluviométricos utilizados neste estudo.

\section{Métodos}

\section{Modelagem hidrossedimentológica}

A produção de sedimento foi calculada por meio da ferramenta de modelagem hidrológica AGWA (Miller et al., 2007), que provê suporte para o funcionamento de todas as fases do modelo hidrológico KINEROS2.

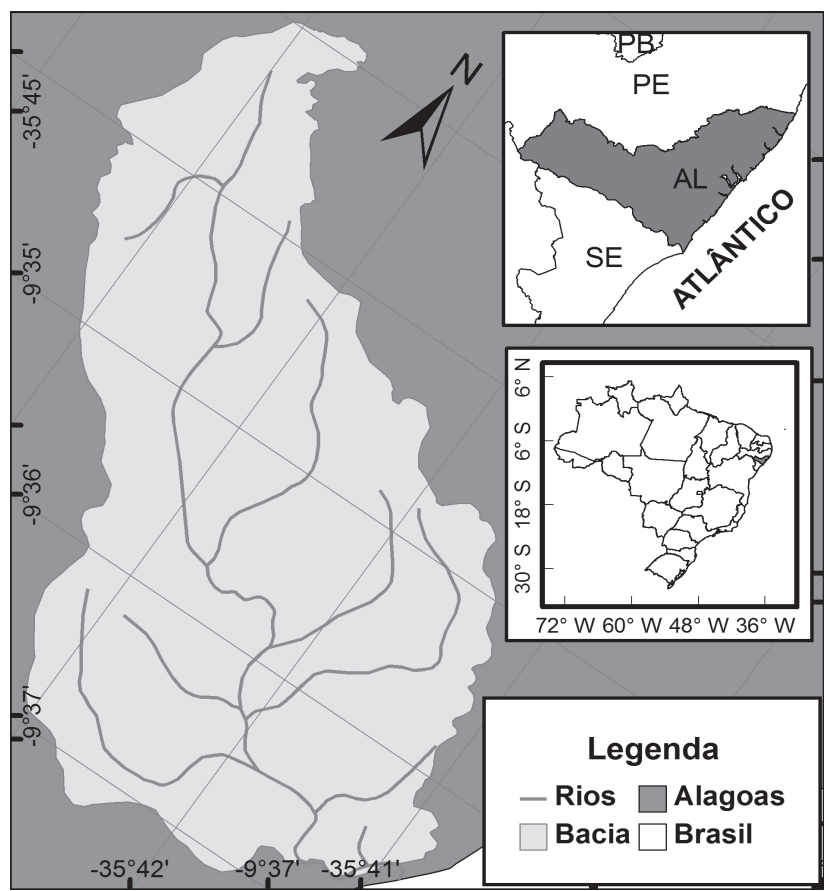

Figura 1. Localização da bacia do rio Jacarecica.
O KINEROS2 é um modelo vazão-erosão usado para estimar o escoamento superficial e a produção de sedimentos em bacias urbanas e rurais. As equações diferenciais que descrevem o transporte de sedimentos e a deposição de sedimentos nos planos e canais são resolvidas pela técnica de diferenças finitas.

\section{Componente de infiltração}

O componente de infiltração utilizado no KINEROS2 permite redistribuição de água no solo, incluindo a recuperação da capacidade de infiltração durante os intervalos entre as chuvas, determinando as taxas de infiltração durante e após esse intervalo sem chuva. A taxa de infiltração $f_{c}$ é uma função da lâmina acumulada de infiltração e de alguns outros parâmetros que descrevem as propriedades de infiltração no solo como: condutividade hidráulica saturada efetiva $K_{s}(\mathrm{~m} / \mathrm{s})$, valor efetivo do potencial de capilaridade $G(\mathrm{~m})$, porosidade do solo $\varphi$ e índice de distribuição dos tamanhos dos poros $\lambda$. Há também um parâmetro opcional $\left(C_{v}\right)$, que descreve a variação aleatória espacial da condutividade hidráulica saturada do solo, assim como um parâmetro que representa a porcentagem de rochas, $R O C K$. O modelo utiliza também uma variável da saturação relativa inicial do solo $S_{i}\left(\mathrm{~m}^{3} / \mathrm{m}^{3}\right)$, cujo valor é dado por $\theta_{\mathrm{t}} / \varphi$, em que $\theta_{i}$ é o índice de umidade inicial do solo. O cálculo da taxa de infiltração $f(c)(\mathrm{m} / \mathrm{s})$ é feito pela seguinte equação (Smith \& Parlange, 1978):

$$
f_{c}=K_{s}\left(1+\frac{\alpha}{e^{\alpha I / B}-1}\right)
$$

em que $B=(G+h)\left(\theta_{s}-\theta_{i}\right)$, combinando os efeitos do potencial efetivo de capilaridade, $G$, e profundidade do fluxo, $h(\mathrm{~m})$, e da capacidade de armazenamento de água do solo, $\Delta \theta=(\theta(s)$ - $\theta(i))$, em que $\theta s$ é a umidade de saturação do solo $\left(\mathrm{m}^{3} / \mathrm{m}^{3}\right)$. O parâmetro $\alpha$ representa o tipo de solo; quando $\alpha$ se aproxima de 0 para areia, a equação 1 aproxima-se à equação de Green-Ampt; e quando $\alpha$ se aproxima de 1 para um solo franco bem uniforme, a equação 1 representa a equação de infiltração de Smith-Parlange (Smith \& Parlange, 1978). Sugere-se que a maioria dos tipos de solo é mais bem representada por um valor de á igual a 0,85.

O valor efetivo do potencial de capilaridade, $G$, é dado pela seguinte expressão:

$$
G=\int_{-\infty}^{0} \frac{K(\psi)}{K_{s}} d \psi
$$

em que $\psi$ é o potencial mátrico do solo (m).

Quadro 1. Distribuição dos postos pluviométricos de interesse do estudo

\begin{tabular}{ccc}
\hline Posto & Latitude & Longitude \\
\hline SEMARH & -9.6215 & -35.6957 \\
INMET & -9.5511 & -35.7678 \\
\hline
\end{tabular}


O modelo de infiltração também leva em consideração a recuperação da capacidade de infiltração do solo, que ocorre em períodos em que não há precipitação, ou em períodos em que a precipitação não produz escoamento, geralmente quando a intensidade de precipitação é inferior à permeabilidade do solo. A equação utilizada no modelo para o cálculo da variação da umidade do solo nesses períodos é:

$$
\frac{d \theta_{o}}{d t}=\frac{\Delta \theta_{i o}}{I}\left[r-K_{i} \cdot\left(K\left(\theta_{o}\right)+\frac{\beta \cdot p \cdot K_{s} \cdot \Delta \theta_{i o} \cdot G\left(\theta_{i}, \theta_{o}\right)}{I}\right)\right]
$$

em que $\Delta \theta_{10}=\theta_{0}-\theta_{\mathrm{i}}$ é a diferença de umidade do solo acima e abaixo da frente de molhamento; $I$, lâmina de infiltração acumulada $(\mathrm{m}) ; r$, taxa de entrada de água na superfície do solo durante a redistribuição de água no solo, que pode ser menor do que a permeabilidade $K s$, negativa (em função da evaporação) ou zero; o parâmetro $\beta$ é um fator de forma dado por:

$$
\beta=\frac{1}{Z\left(\theta_{o}-\theta_{i}\right)} \int_{0}^{z}\left(\theta-\theta_{i}\right) d z=0,85
$$

em que $Z$ é a profundidade da frente de molhamento; $p$, um fator efetivo de profundidade ( $p=2$ para $r=0$; $p=1,5$ para $0<r<K s ; p=3$ para $r<0)$; e $G\left(\theta_{i}, \theta_{0}\right)$, o valor efetivo do potencial de capilaridade na frente de redistribuição de água (m).

Nos períodos em que a umidade do solo está abaixo da umidade de saturação, a equação utilizada para calcular a condutividade hidráulica do solo é (Brooks \& Corey, 1964):

$$
K(\theta)=K\left(\frac{\theta-\theta_{r}}{\theta_{s}-\theta_{r}}\right)^{3+\frac{2}{\lambda}}
$$

em que $\theta$ é a umidade do solo no período de redistribuição de água; $K_{s}$, a permeabilidade efetiva do solo $(\mathrm{m} / \mathrm{s}) ; \theta_{\mathrm{r}}$, a umidade residual do solo; $\theta_{\mathrm{s}}$, a umidade do solo na saturação; e $\lambda$, o parâmetro de distribuição do tamanho dos poros.

Maiores detalhes sobre o modelo de redistribuição de água no solo, utilizado no modelo KINEROS2, podem ser obtidos em Smith et al. (1993) e Corradini et al. (1994).

\section{Modelagem da erosão nos planos e canais}

A equação geral que descreve a dinâmica dos sedimentos dentro do fluxo é a de balanço de massa, similar àquela para o fluxo de água (Bennett, 1974):

$$
\frac{\partial\left(A C_{s}\right)}{\partial t}+\frac{\partial\left(Q C_{s}\right)}{\partial x}-e(x, t)=q_{s}(x, t)
$$

em que $C_{s}$ é a concentração de sedimento no fluxo $\left(\mathrm{m}^{3} / \mathrm{m}^{3}\right) ; Q$, vazão hidráulica $\left(\mathrm{m}^{3} / \mathrm{s}\right) ; A$, área da seção transversal do plano ou canal $\left(\mathrm{m}^{2}\right) ; e$, taxa de erosão do solo $\left(\mathrm{m}^{2} / \mathrm{s}\right)$; e $q_{s}$, taxa de entrada lateral de sedimento no fluxo nos canais $\left(\mathrm{m}^{3} / \mathrm{s} / \mathrm{m}\right)$.
Nos planos, a erosão é modelada por dois componentes principais: pelo impacto das gotas de chuva no solo e pela erosão (ou deposição) hidráulica, que são resultados da interação entre a força de cisalhamento do fluxo e a tendência das partículas de solo no fluxo ao se depositarem sob efeito da força da gravidade. A erosão resultante é o somatório da taxa da erosão provocada pelo impacto das gotas de chuva $e_{s}$ e da erosão hidráulica $e_{h}$ :

$$
e=e_{s}+e_{h}
$$

A erosão por impacto das gotas de chuva é estimada pela seguinte expressão (Meyer \& Wischmeier, 1969):

$$
e_{s}=c_{f} e^{-c} h^{h} i^{2}
$$

em que $i$ é a intensidade da precipitação $(\mathrm{m} / \mathrm{s}) ; c_{f}$, um coeficiente a ser determinado experimentalmente ou por calibração, que está relacionado às propriedades do solo e da superfície; e $e^{-c} h^{h} i^{2}$, um fator que representa a redução na erosão por impacto das gotas de chuva por causa do aumento da lâmina d'água. $\mathrm{O}$ parâmetro $c_{h}$ representa a efetividade do amortecimento da superfície da água, fixado no modelo como sendo igual a 656 . A erosão hidráulica $\left(e_{h}\right)$ é estimada como sendo linearmente dependente da diferença entre a capacidade de transporte do fluxo (concentração de equilíbrio) e a concentração real de sedimento e é dada pela equação:

$$
e_{h}=c_{g}\left(C_{m}-C_{s}\right) A
$$

em que $c_{m}$ é a concentração de equilíbrio na capacidade de transporte; $c_{s}=c_{s}(x, t)$, a concentração real de sedimentos no fluxo; e $c_{g}$, um coeficiente da taxa de transferência de sedimentos $\left(\mathrm{s}^{-1}\right)$, calculado por:

$$
\begin{aligned}
C_{s} & =C_{0} \frac{v_{S}}{h} \text { se } C_{s} \leq C_{m}(\text { erosão }) \text { ou } \\
C_{g} & =\frac{v_{s}}{h} \text { se } C_{s}>C_{m}(\text { deposição })
\end{aligned}
$$

em que $c_{o}$ é um coeficiente que reflete a coesão do solo; e $v_{s}$, a velocidade de queda da partícula $(\mathrm{m} / \mathrm{s})$.

O modelo KINEROS utiliza a fórmula de capacidade de transporte de Engelund \& Hansen (1967) com a inclusão de um limite crítico do valor da potência unitária do fluxo $\Omega=u S$ (Unit Stream Power) igual a $0,004 \mathrm{~m} / \mathrm{s}$, em que $u$ é a velocidade do fluxo $(\mathrm{m} / \mathrm{s})$; e $S$, a declividade, para estender a sua aplicabilidade a fluxos rasos. A equação para o cálculo da concentração de sedimentos na capacidade de transporte é a seguinte:

$$
c_{m}=\frac{0,05}{d\left(S_{s}-1\right)^{2}} \sqrt{\frac{S^{*} h}{g}(\Omega-0,004)}
$$

em que $g$ é a aceleração da gravidade $\left(\mathrm{m} / \mathrm{s}^{2}\right) ; S_{s}$, a densidade relativa dos sólidos, igual a 2,$65 ; d$, o diâmetro do sedimento (m); e $h$, a profundidade do fluxo (m); as outras variáveis já foram definidas anteriormente.

A velocidade de queda da partícula é calculada pela seguinte equação: 


$$
v_{s}^{2}=\frac{4}{3} \frac{g\left(S_{s}-1\right) d}{C_{D}}
$$

em que $C_{D}$ é o coeficiente de arrasto da partícula, que é uma função do número de Reynolds, e é calculado pela seguinte expressão:

$$
C_{D}=\frac{24}{R_{n}}+\frac{3}{\sqrt{R_{n}}}+0,34
$$

em que $R n$ é o número de Reynolds, calculado como $R n=v_{s} d / \mathrm{y}$, em que y é a viscosidade cinemática da água. A velocidade de queda da partícula é encontrada resolvendo simultaneamente as equações 12 e 13 .

A simulação do transporte de sedimentos para os canais é realizada de maneira semelhante à simulação do transporte de sedimentos nos planos. A principal diferença nas equações é que a erosão por impacto das gotas de chuva é desprezada, e o termo $q_{s}$ torna-se importante na representação da entrada de fluxo lateral.

\section{Calibração do modelo}

A bacia do rio Jacarecica foi discretizada em 85 elementos, sendo 52 planos e 21 canais. Cada elemento plano foi definido, levando-se em consideração a homogeneidade das características dos solos, da declividade média, e das linhas de fluxo. Alguns dos parâmetros do modelo exercem maior influência sobre os processos de erosão, como os valores da condutividade hidráulica (Ks), capilaridade $(\mathrm{G})$ e porosidade $(\Phi)$. Neste estudo, os valores de G, Ks e $\Phi$ foram modificados com base em Silva \& Santos (2008); a calibração foi ajustada até que o volume escoado calculado se aproximasse do volume escoado observado, utilizando o método da tentativa e erro.

Neste trabalho, os valores dos parâmetros foram estabelecidos por meio do AGWA, utilizando-se as tabelas da FAO relacionadas com o tipo de solo; a determinação dos valores de parâmetros ficou a cargo do modelo AGWA, resultando nos dados listados no quadro 2.

\section{RESULTADOS E DISCUSSÃO}

\section{Modelagem da produção de sedimentos}

Após a calibração do modelo, foi estimada a produção de sedimentos da bacia do rio Jacarecica,

Quadro 2. Valores dos parâmetros usados no estudo

\begin{tabular}{lccc}
\hline Parâmetro & Símbolo & Plano & Canal \\
\hline Capilaridade média do solo & $\mathrm{G}$ & 272,73 & 101 \\
Condutividade hidráulica & $\mathrm{Ks}$ & 4,76 & 210 \\
Porosidade & $\Phi$ & 0,44 & 0,44 \\
\hline
\end{tabular}

para o ano de 2010. Observa-se que a maior produção de sedimentos gerada na bacia está associada aos meses com precipitação mais elevada (Quadro 3). Nos meses em que a precipitação média mensal foi baixa (outubro, novembro e dezembro), a produção média mensal de sedimentos também foi bem inferior à dos demais meses.

A relação entre a precipitação pluvial média mensal e a produção de sedimentos mensal calculada está indicada na figura 2 . O coeficiente de determinação $\left(R^{2}=0,97\right)$ foi satisfatório. Percebe-se que a produção de sedimentos no exutório da bacia variou entre 0 e 120 t/ha/mês, para uma variação de chuva da ordem de 13 a $533 \mathrm{~mm} / \mathrm{mês}$; o mês de junho apresentou disparidade em relação aos demais meses, com valor mensal médio de sedimentos elevado, em razão principalmente da alta precipitação ocorrida nesse mês.

A produção total de sedimentos alcançou um total de $130 \mathrm{t}$, para um total precipitado de $1.891,2 \mathrm{~mm}$. Salienta-se que esse total precipitado foi bem acima da média climatológica para o município de Maceió,

Quadro 3. Produção de sedimentos calculada no exutório da bacia, para o ano de 2010

\begin{tabular}{lcc}
\hline \multicolumn{1}{c}{ Mês } & Precipitação pluvial & Sedimento \\
\hline & $\mathrm{mm} / \mathrm{mês}$ & $\mathrm{t} / \mathrm{ha} / \mathrm{mês}$ \\
Janeiro & 189,3 & 6,57 \\
Fevereiro & 144,2 & 1,34 \\
Março & 119,8 & 0,05 \\
Abril & 140,6 & 0,90 \\
Maio & 133,8 & 0,50 \\
Junho & 532,8 & 120 \\
Julho & 226,2 & 0,01 \\
Agosto & 148,8 & 1,50 \\
Setembro & 118,0 & 0,02 \\
Outubro & 105,0 & 0 \\
Novembro & 12,6 & 0 \\
Dezembro & 20,1 & 0 \\
\hline
\end{tabular}

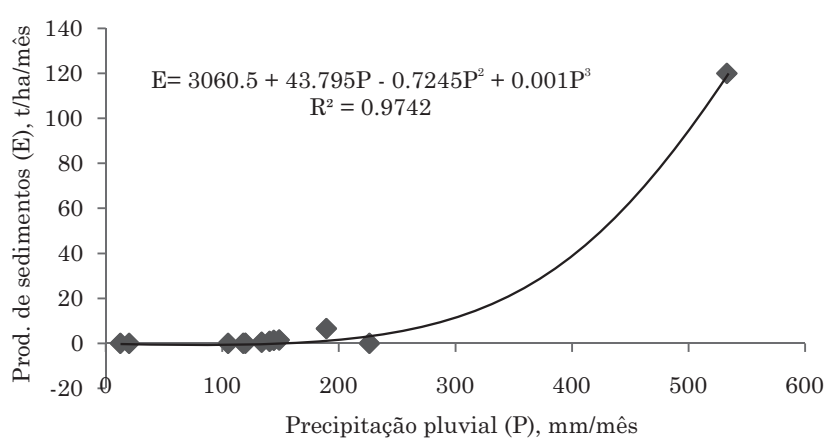

Figura 2. Relação entre a precipitação pluvial média mensal e a produção de sedimentos mensal calculada. 
tendo os meses de janeiro, fevereiro e junho com a maior produção de sedimentos ao mês.

Os mapas, a seguir, apresentam a espacialização da produção de sedimentos para cada plano e canal discretizados da bacia, a partir da precipitação mensal observada para o período compreendido entre janeiro e junho de 2010 e implantados no SIG, para a representação espacial da estimativa da produção de sedimentos.

Pode-se observar que as áreas com menor produção de sedimentos na bacia são as com vegetação predominante, principalmente no nordeste da parte baixa da bacia.

Nota-se que os planos com mais produção de sedimentos se encontram nas partes alta e média da bacia, onde já foram identificadas áreas com grande potencial de produção de sedimento. Já no sudoeste da parte baixa da bacia, região com urbanização bem acentuada, também são encontrados maiores valores de produção de sedimentos, evidenciando a relação entre áreas urbanizadas e a produção de sedimentos.

Melo (2010) concluiu que a utilização do modelo KINEROS2 utilizado pela interface AGWA2, sem calibração dos parâmetros, se evidenciou alternativa interessante para ser implementada, já que se trata de um modelo robusto em uma interface mais simples e com uma entrada de dados prática, pois por meio dessa interface é possível utilizar informações contidas em dados vetoriais geoespaciais obtidas em base de dados internacionais.

Na bacia do córrego do Capão Comprido, no DF, Lopes (2010) concluiu que, em relação aos resultados,
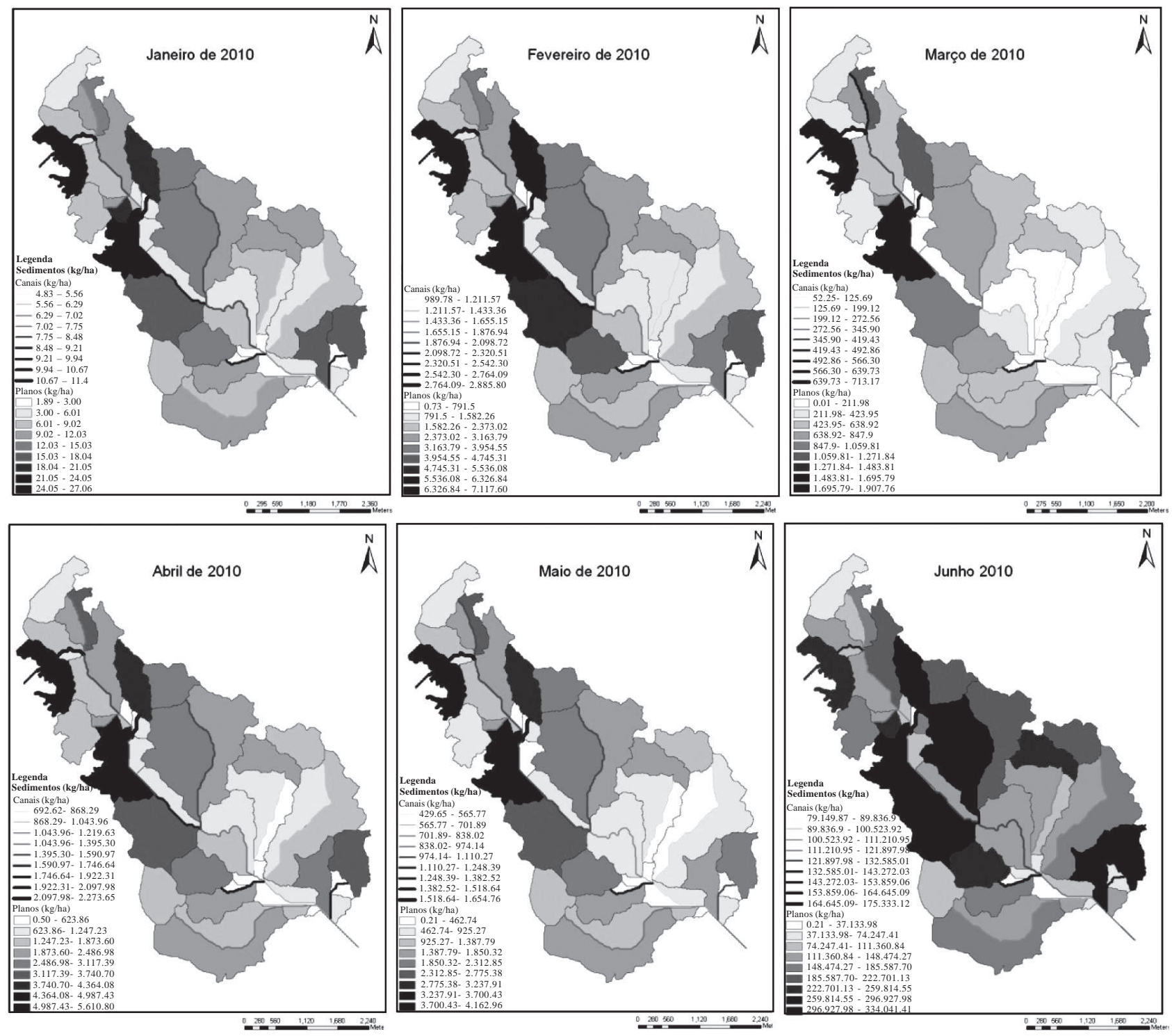

Figura 3. Espacialização da produção média calculada de sedimentos para a bacia do rio Jacarecica, de janeiro a junho de 2010. 

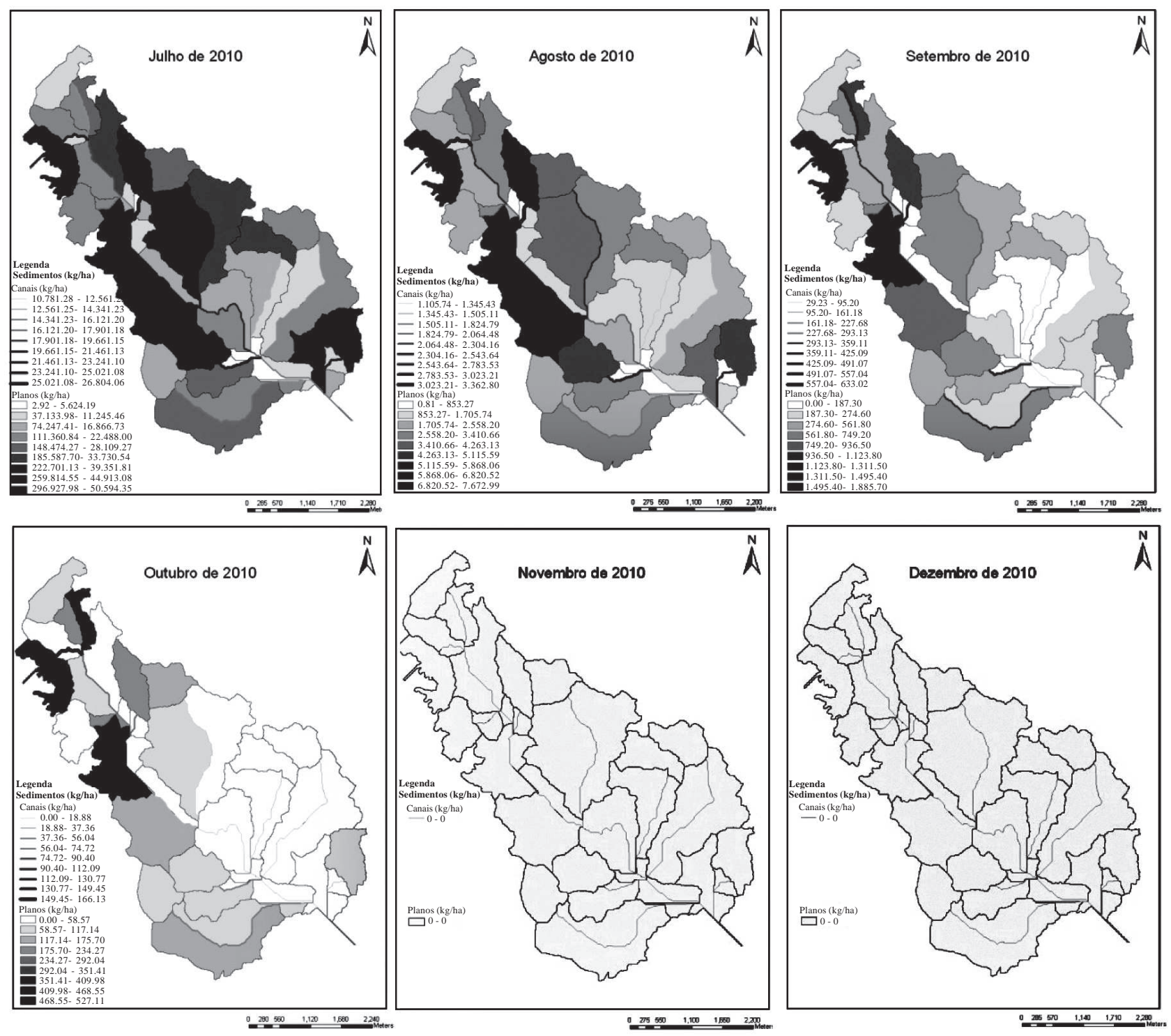

Figura 4. Espacialização da produção média calculada de sedimentos para a bacia do rio Jacarecica, de julho a dezembro de 2010.

apesar de não ter havido a calibração do modelo, para alguns eventos, os resultados apresentaram-se coerentes com os obtidos anteriormente para bacia. Foi necessária a adaptação de alguns dados de entrada que represente a realidade da região, além da calibração do modelo para que os resultados do KINEROS2 possam ser utilizados para avaliação quantitativa da região.

A figura 4 ilustra a espacialização da produção de sedimentos para cada plano e canal discretizados da bacia, a partir da precipitação mensal observada para o período compreendido entre julho e dezembro de 2010, e implantados no SIG.

Os meses de outubro, novembro e dezembro tiveram produção de sedimentos insignificantes pelo modelo, pois o índice pluviométrico foi menor do que os demais meses. No que tange à identificação das áreas susceptíveis ao processo de erosão, os resultados evidenciaram que as áreas localizadas na parte noroeste da bacia foram as que mais contribuíram para o processo de erosão, se comparadas com os planos inseridos na parte sul da bacia, o que pode ser explicado pelo fato de as maiores declividades se localizarem na parte noroeste da bacia. Pode-se observar que as áreas que apresentam menos produção de sedimentos na bacia são as com vegetação predominante, localizadas principalmente na parte nordeste da parte baixa da bacia.

Neste estudo, o modelo representou bem a bacia, diferente do observado por Lopes (2003), o qual, após extenso estudo utilizando o modelo KINEROS2 em uma pequena bacia na região semiárida da Paraíba, afirmou que, apesar da robustez, o modelo em questão não consegue representar bem a produção de 
sedimentos na bacia estudada, já que o intervalo do parâmetro que representa a erosão causada pelo impacto da gota de chuva deveria ter intervalo de variação menor, para melhor discretização em cada tipo de cobertura vegetal.

Silva \& Santos (2008), aplicando o modelo KINEROS acoplado a um SIG, para a bacia do rio Pirapama, com cerca de $600 \mathrm{~km}^{2}$, localizada na zona litorânea do Estado de Pernambuco, estimam que a produção de sedimentos atingiu valores maiores que 200 t/ano.

\section{CONCLUSÕES}

1. A influência da urbanização e da exposição do solo nos processos de produção de sedimentos ao longo da bacia foi bem evidenciada na escala espaço-temporal para o ano analisado, verificando-se que os planos com esses tipos de uso do solo produzem mais sedimentos no decorrer do ano. Já a vegetação predominante, principalmente na parte baixa da bacia, minimiza a produção de sedimentos.

2. O modelo KINEROS 2, acoplado ao AGWA2, representou razoavelmente a distribuição espacial da produção de sedimentos na bacia do rio Jacarecica, bacia essa de pequena escala espacial, que pode ser útil na identificação de áreas suscetíveis e em processo de erosão.

3. A perda de solo potencial média na bacia Jacarecica foi estimada em aproximadamente $130 \mathrm{Mg} /$ ha/ano, que deve ser considerada alta, pois se encontra entre 50 e $200 \mathrm{Mg} / \mathrm{ha} / \mathrm{ano}$, segundo a classificação proposta pela FAO (1980).

\section{LITERATURA CITADA}

ARAÚJO, J.C. \& KNIGHT, D.W. A review of the measurement on sediment yield in different scales. R. Escola Minas, 58:257-265, 2005.

BENNETT, J.P. Concepts of mathematical modeling of sediment yield. Water Resour. Res., 10:485-492, 1974.

BROOKS, R.H. \& COREY, A.T. Hydraulic properties of porous media. Fort Collins, Colorado State University, 1964. (Hydrology Paper, 3)

CABRAL, S.L. Avaliação dos processos sedimentológicos na bacia do rio Jacarecica, em Maceió-AL. Maceió, Universidade Federal de Alagoas, 2011. 130p. (Dissertação de Mestrado)

CORRADINI, C.; MELONE, F. \& SMITH, R.E. Modeling infiltration during complex rainfall sequences. Water Resour. Res., 30:2777-2784, 1994.
ENGELUND, F. \& HANSEN, E. A monograph on sediment transport in alluvial streams. Copenhagen, Teknisk Vorlag, 1967.62p.

FOOD AND AGRICULTURE ORGANIZATION - FAO. La erosión del suelo por el agua: Algunas medidas para combatirla en las tierras de cultivo. Roma, 1980. 207p. (Cuadernos de fomento agropecuário de la Organización de Las Naciones Unidas, 81)

FIGUEIREDO, E.E. \& BATHURST, J.C. Runoff and sediment yield predictions in a semiarid region of Brazil using SHETRAN. In: IAHS DECADE ON PREDICTION IN UNGAUGED BASINS, HYDROLOGICAL SCIENCES, 2002, Brasília. Resumos... Brasília, 2002. CD-ROM

FIROUZABADI, P.Z. \& DAVOODI, A. Study on soil erosion and sedimentation in Alashtar watershed using image processing software. In: ISPRS CONGRESS, HYDROLOGICAL SCIENCES, 2004, Istanbul. Resumos... Istanbul, 2004. CD-ROM

LOPES, W.T.A. Efeitos de escala na modelagem hidrossedimentológica na região semi-árida da Paraíba. Campina Grande, Universidade Federal da Paraíba, 2003. 196p. (Dissertação de Mestrado)

LOPES, G.R. Estudos hidrológicos e hidrossedimentológicos na bacia do córrego do Capão Comprido, DF. Brasília, Universidade de Brasília, 2010. 123p. (Dissertação de Mestrado) (Publicação PTARH. DM-131/2010)

MELO, H.A. Integração entre modelos hidrológicos e sistemas de informações geográficas: Uma avaliação metodológica. Campina Grande, Universidade Federal de Campina Grande, 2010. 106p. (Dissertação de Mestrado)

MEYER L.D. \& WISCHMEYER, W.H. Mathematical simulation of the process of soil erosion by water. Trans. ASAE, 12:754-758, 1969.

MILLER, S.N.; GOODRICH, D.C.; HERNANDEZ, M.; SEMMENS, D.J.; MILLER, R.C.; KEPNER, W.G. \& GUERTIN, D.P. The automated geospatial watershed assessment tool. Environ. Model. Software, 22:365-377, 2007.

PAIVA, E.M.C. \& PAIVA, J.B.D. Hidrologia aplicada à gestão de pequenas bacias hidrográficas. Porto Alegre, ABRH, 2003. 625p.

SILVA, R.M. \& SANTOS, C.A.G. Estimativa da produção de sedimentos mediante uso de um modelo hidrossedimentológico acoplado a um SIG. R. Bras. Eng. Agríc. Amb., 12:520-526, 2008.

SMITH, R.E. \& PARLANGE, J.Y. A parameter-efficient hydrologic infiltration model. Water Resour. Res., 14:533538, 1978.

SMITH, R.E.; CORRADINI, C. \& MELONE, F. Modeling infiltration for multistorm runoff events. Water Resour. Res., 29:133-144, 1993. 